hep-th/9708147

PUPT-1718

RU-97-68

\title{
Brane Dynamics From the Born-Infeld Action
}

\author{
Curtis G. Callan, Jr. ${ }^{1}$ \\ Joseph Henry Laboratories, Princeton University, Princeton, NJ 08544 \\ and \\ J.M. Maldacena ${ }^{2}$ \\ Physics Department, Rutgers University, Piscataway NJ 08855
}

\begin{abstract}
We use the abelian Born-Infeld action for the worldvolume gauge field and transverse displacement scalars to explore new aspects of D-brane structure and dynamics. We study several classic gauge field configurations, including point charges in any worldvolume dimension and vortices in two worldvolume dimensions, and show that, with an appropriate excitation of the transverse coordinate field, they are BPS-saturated solutions. The Coulomb point charge solutions turn out to represent, with considerable fidelity, fundamental strings attached to the brane (their magnetic counterparts describe D1-branes attached to D3-branes). We also show that S-matrix for small excitations propagating on the point charge solution is consistent with (and gives further illuminating information about) Polchinski's effective open string boundary condition.
\end{abstract}

August 1997

\footnotetext{
1 e-mail: callan@viper.princeton.edu

2 e-mail:malda@physics.rutgers.edu
} 


\section{Introduction}

The Born-Infeld action for D-brane dynamics is a $p+1$-dimensional $U(1)$ gauge theory with $9-p$ neutral scalars describing transverse fluctuations of the brane. This action is the dimensional reduction of 10-dimensional supersymmetric Born-Infeld electrodynamics [1,2]. For a variety of reasons, most treatments of this system have been based on a linear, Maxwellian, approximation to this nonlinear action. In this paper we will take the specific nonlinearities of the BI action seriously, revealing some interesting new insights into the statics and the dynamics of intersecting branes. On the static side, we will show that fundamental strings attached to branes are described by Coulomb point charge solutions of Born-Infeld electrodynamics. More elaborate brane intersections correspond to other localized gauge objects such as the magnetic monopole and the vortex line. Some of the essential features of the energetics of these solutions come from supersymmetry and BPS arguments, of course, but the dynamics rely on the specific nonlinear properties of the action. This is particularly true of the S-matrix describing the way small fluctuations on one brane can 'leak through' to the other brane. This S-matrix determines the effective boundary condition one of the branes provides for the worldsheet field theory on the other. Its study provides interesting insights into Polchinski's open string theory definition of D-branes.

In what follows, we will explain these results in detail, commenting as we go on their reliability and their implications for other aspects of string theory and M-theory.

\section{Strings from $U(1)$ point charges: A Linear Argument}

We begin by using BPS arguments to find some solutions of the p-brane worldsheet gauge theory which have the interpretation of strings ending on branes and D-p branes ending on $\mathrm{D}-(\mathrm{p}+2)$-branes. Let us first assume that the massless excitations of a p-brane are described by the dimensional reduction of the 10-dimensional Maxwell action. This ignores important nonlinearities (which we will consider later) but will provide a useful orientation. The supersymmetry variation of the gaugino is

$$
\delta \chi=\Gamma^{\mu \nu} F_{\mu \nu} \epsilon
$$

where $\mu \nu$ are ten-dimensional indices. A BPS background is one where $\delta \chi=0$ for some $\epsilon$. Now imagine we have a point charge Coulomb gauge field, or $A_{0}=c_{p} / r^{p-2}$ where $r$ is the spatial $p$-dimensional distance and $c_{p}$ is fixed by some charge quantization condition. So far only $F_{0 r} \neq 0$ and (2.1) shows that there are no preserved supersymmetries since $\Gamma^{0 r}$ has no zero eigenvalues. We can, however, also excite one of the transverse scalar 
fields. Indeed, $X^{9}=c_{p} / r^{p-2}$ is a solution of the linearized field equation for $X^{9}$ for which $F_{9 r} \equiv \partial_{r} X^{9}=F_{0 r}$. In the presence of both excitations (2.1) reduces to

$$
\left(\Gamma^{0 r}+\Gamma^{9 r}\right) \epsilon=0 \Rightarrow\left(\Gamma^{0}+\Gamma^{9}\right) \epsilon=0 .
$$

The usual arguments tell us that half of all choices for $\epsilon$ satisfy this condition. In other words, this background preserves half the supersymmetries originally possessed by the p-brane and is still a BPS-saturated state.

What is the interpretation of such a state? The excitation of the $X^{9}$ field that we have chosen amounts to giving the brane a transverse 'spike' protruding in the $\hat{9}$ direction and running off to infinity. It turns out that this spike must be interpreted as a fundamental string attached to the D-p-brane. To show this, we calculate the energy of the configuration. Of course, the energy of a Coulomb field is strictly infinite, but let us look at this infinity in more detail by calculating the energy for $r \geq \delta$ where $\delta$ is small. We find 1

$$
E(\delta)=\frac{1}{(2 \pi)^{p} g} \int d^{p} r \frac{1}{2}\left\{F_{0 r}^{2}+F_{09}^{2}\right\}=\frac{c_{p}^{2}(p-2)^{2}}{(2 \pi)^{p} g} \int d^{p} r \frac{1}{r^{2(p-1)}}=\frac{c_{p}^{2}(p-2) \Omega_{p-1}}{(2 \pi)^{p} g} \frac{1}{\delta^{p-2}},
$$

where $\Omega_{p}$ is the volume of the unit $p$-sphere. Remarkably, this energy is proportional to the value of $X^{9}$ at $\delta$ :

$$
E(\delta)=\frac{c_{p} \Omega_{p-1}(p-2)}{(2 \pi)^{p} g} X^{9}(\delta)
$$

In the limit $\delta \rightarrow 0$ the transverse 'spike' is infinitely long and has infinite energy by virtue of having a constant energy per unit length. This interprets the Coulomb energy divergence as being due to an infinitely long string attached to the brane! We should be able to tell just what kind of string it is from the value of its energy per unit length.

Since we expect the source of a worldvolume electric field to be the fundamental string, we should be able to see that (2.3) matches the energy per unit length of a fundamental string. To do this, we need to identify the charge quantization constant $c_{p}$. Consider first a 1-brane with (necessarily constant) gauge field $F_{01}$ excited. Depending on the strength of $F_{01}$, this is a type IIB D-string with some number $n$ of adsorbed fundamental strings. From the BPS mass formula we know that the tension of such excited strings is $\left(1 / g+g n^{2} / 2\right) T_{f}$ for small $n$ and string coupling $g$ (where $T_{f}=1 / 2 \pi$ is the fundamental string tension). In the linearized weak field limit of the Born-Infeld action the additional tension due to the electric field is $(1 / 2 g) F_{01}^{2} T_{f}$. This implies that the quantization condition on the onedimensional electric field is $F_{01}=g n$. The higher-dimensional cases are related to this by T-dualities and one can see that the general quantization condition on the electric flux is

$$
\frac{1}{(2 \pi)^{p-1}} \int_{S_{p-1}} F_{0 r}=g n
$$

1 We are setting $\alpha^{\prime}=1$ and using conventions in which $g \rightarrow 1 / g$ under S-duality. 
This implies that $c_{p}(p-2) \Omega_{p-1} /(2 \pi)^{p-1}=g$ and that the energy of the unit charge Coulomb solution is

$$
E(\delta)=\frac{1}{2 \pi} X^{9}(\delta)=T_{f} X^{9}(\delta)
$$

This is the energy of a fundamental string of length $X^{9}(\delta)$. The analysis works for any value of $p$ and tells us that there is a BPS gauge configuration describing the attachment of a fundamental string to any D-brane. This is exactly what Polchinski's string theoretic description of D-brane solitons would lead us to expect [1].

There is one further possibility for solutions of the kind we are discussing. On a 3+1-dimensional worldvolume (D3-brane) there exist magnetic point charge solutions for which $F_{\hat{\theta} \hat{\phi}}=N c_{m} / r$ (and $c_{m}$ will be determined by magnetic monopole charge quantization conditions)2. Taking the transverse coordinate excitation to be $X^{9}=N c_{m} / r$, the condition for unbroken susy now reads

$$
\left(\Gamma^{\hat{\theta} \hat{\phi}}+\Gamma^{9 r}\right) \epsilon=0 \quad \text { or } \quad \Gamma^{1239} \epsilon=\epsilon .
$$

This is exactly what we expect for the preserved susy when a type IIB D1-brane along $\hat{9}$ is attached to a D3-brane along 123. The energy analysis of this solution is consistent with this picture and shows that $c_{m}=\pi$, independent of the string coupling $g$. Among other things, this implies that the shape of the solution is independent of the string coupling. Clearly the same solution would describe a D-p-brane ending on a D- $(p+2)$-brane.

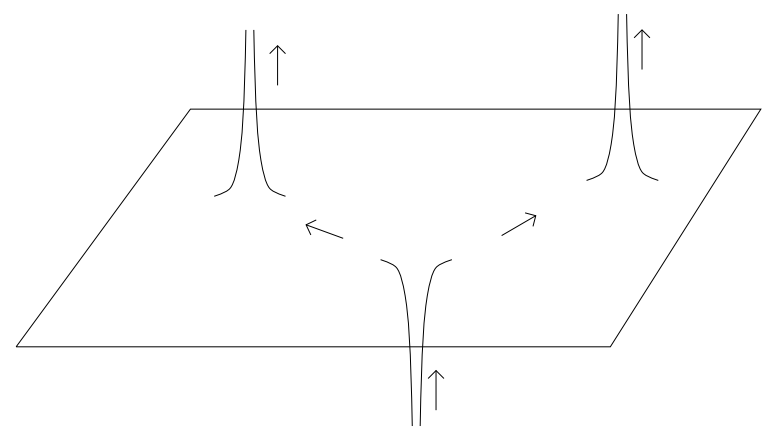

FIGURE 1: Branes with multiple strings attached on both sides.

The arrows represent the direction of the electric field, which also defines the orientation of the string

It is interesting that in any of the above solutions we may replace the single-center Coulomb solution with a completely general solution of Laplace's equation:

$$
1 / r^{p-2} \rightarrow \sum \pm \frac{1}{\left|\vec{r}-\vec{r}_{i}\right|^{p-2}}
$$

2 Were $\hat{\theta}, \hat{\phi}$ denote unit vectors. 
where for each $i$ we have a choice of signs. When this function is used to specify $X^{9}$, we see that it represents multiple strings ending at arbitrary locations on a brane (see Figure 1). The two signs of the charge correspond to strings attached to different sides of the brane, but having the same orientation. We could say that strings with positive signs in (2.8) are ending on the brane while the ones with negative sign are 'departing' from the brane. If we had strings of different orientations the solution would not be BPS. A careful analysis shows that the energy is independent of the locations of the attachment points

$$
E=\sum_{i}\left|X^{9}\left(\delta_{i}\right)\right| T_{f}
$$

and is exactly sum of the lengths of the various strings times the fundamental string mass per unit length. An important point is that there is no extra constant term to be identified as an 'attachment energy'.

Note that this type of solution allows us to describe the deformation of a fundamental string traversing the brane into two open strings ending on the brane at separate points. It might seem paradoxical that two solitons with opposite charges are not atracted to each other. What happens is that they have also a scalar charge (associated to $X^{9}$ ) and two opposite scalar charges repel each other. In other words: the Coulomb energy is exactly balanced by the elastic deformation energy of the brane. In short, we now have a BPS description of the breaking of a fundamental closed string into two open strings when it meets a D-brane. Notice that the same description applies to a D1-brane meeting a D3-brane.

There are implications for M-theory to be drawn from this as well. Consider a D4 brane intersecting fundamental type IIA strings as above. This should be the same as the $X^{11}$ compactification of the M-theory membrane ending on an M-theory fivebrane along a line. Since the IIA D-fourbrane action is the dimensional reduction of the M-fivebrane action we can 'lift' the solutions we have found to solutions of the M-fivebrane action that are invariant along $X^{11}$. This is indeed the solution describing an M-twobrane ending on a fivebrane and the intersection is one dimensional and is oriented along $X^{11}$. After the usual redefinition of parameters, the quantization conditions work out exactly right. From what we now know about how the D4-brane can 'slice' fundamental strings, we learn that when an M-theory membrane intersects a fivebrane on a line, the membrane can break into open membranes attached to the fivebrane, which can then separate and move independently [3, 团. This motion is a deformation respecting the BPS condition, a property which has been used to calculate the entropy of four-dimensional black holes [5]. 


\section{Strings from point charges: Non-Linear Treatment}

In the previous section we made use of the properties of the quadratic, Maxwellian, limit of the action for the worldsheet degrees of freedom of D-branes. Insofar as we are interested in the properties of strict BPS states (state counting and masses), we probably can't go wrong in doing so. However, as soon as we interest ourselves in non-BPS states, no matter how close in energy to the BPS limit, the Maxwellian action gives, as we shall see, qualitatively incorrect results. Sooner or later, therefore, we will be obliged to redo the calculations of the previous section using the fully nonlinear BI action. That will be the topic of this section.

Pure Born-Infeld $U(1)$ electromagnetism in ten dimensions is described by the action

$$
L_{10}=-\frac{1}{(2 \pi)^{9} g} \int d^{10} x \sqrt{\operatorname{det}(1+F)}
$$

where $F_{\mu \nu}$ is the gauge field strength. To reduce this to the dynamics of a $p$-brane, identify $A_{i}$ for $i=0,1, \ldots, p$ with the worldbrane gauge field and $A_{a}$ for $a=p+1, \ldots, 9$ with the transverse displacements of the brane (and only allow fields to depend on the worldbrane coordinates $x^{i}$ ) [1]. We won't attempt a completely general analysis of this system, but will focus on one or two instructive special cases.

First, consider the case where the worldbrane gauge field is purely electric and only one transverse coordinate (call it $X$ ) is excited. In that case, the worldbrane action reduces to

$$
L=-\frac{1}{g_{p}} \int d^{p} x \sqrt{\left(1-\vec{E}^{2}\right)\left(1+\vec{\nabla} X^{2}\right)+(\vec{E} \cdot \vec{\nabla} X)^{2}-\dot{X}^{2}} .
$$

The normalization corresponds to the background tension of a D-p-brane in its ground state (hence the factor of $1 / g_{p}$, where we have defined $g_{p} \equiv g(2 \pi)^{p}$ ). Since we are going to want the energy function anyway, it is best to pass to the Hamiltonian formalism right away. The canonical momenta associated with $\vec{A}_{i}$ and $X$, respectively, are

$$
\begin{aligned}
& g_{p} \vec{\Pi}=\frac{\vec{E}\left(1+\vec{\nabla} X^{2}\right)-\vec{\nabla} X(\vec{E} \cdot \vec{\nabla} X)}{\sqrt{\left(1-\vec{E}^{2}\right)\left(1+\vec{\nabla} X^{2}\right)+(\vec{E} \cdot \vec{\nabla} X)^{2}-\dot{X}^{2}}} \\
& g_{p} P=\frac{\dot{X}}{\sqrt{\left(1-\vec{E}^{2}\right)\left(1+\vec{\nabla} X^{2}\right)+(\vec{E} \cdot \vec{\nabla} X)^{2}-\dot{X}^{2}}}
\end{aligned}
$$

and, with a little algebra, one can construct the Hamiltonian:

$$
H=\frac{1}{g_{p}} \int d^{p} x \sqrt{\left(1+\vec{\nabla} X^{2}\right)\left(1+g_{p}^{2} P^{2}\right)+g_{p}^{2} \vec{\Pi}^{2}+g_{p}^{2}(\vec{\Pi} \cdot \vec{\nabla} X)^{2}} .
$$

The canonical momentum $\vec{\Pi}$ is of course subject to the constraint $\vec{\nabla} \cdot \vec{\Pi}=0$ (this follows from the absence of a canonical momentum for $A_{0}$ ) and we can use this to turn (3.4) into 
an action for the transverse displacement alone. (Since we assumed that the magnetic field vanishes, we have to be careful not to look for solutions that violate this condition.)

Our primary goal is to reproduce the configurations described in the previous section, so we shall first look for static solutions. We first solve the constraint by setting

$$
g_{p} \vec{\Pi}=\vec{\nabla} \Lambda, \quad \nabla^{2} \Lambda=0 \Rightarrow \Lambda=\Sigma_{i} \frac{q_{i}}{\left|\vec{r}-\vec{r}_{i}\right|^{p-2}}
$$

where the charges $q_{i}$ are the same as the ones in the last section. Since the charge quantization condition is a long range property, far from the center we can apply the usual weak field charge quantization conditions. The static equation for $X$, gotten by varying (3.4) after setting $P=0$ and constraining $\Pi$, is

$$
\vec{\nabla} \cdot\left[\frac{\vec{\nabla} X+g_{p}^{2} \vec{\Pi}(\vec{\Pi} \cdot \vec{\nabla} X)}{\sqrt{1+\vec{\nabla} X^{2}+g_{p}^{2} \vec{\Pi}^{2}+g_{p}^{2}(\vec{\Pi} \cdot \vec{\nabla} X)^{2}}}\right]=0 .
$$

We now observe that if we set $\vec{\nabla} X=g_{p} \vec{\Pi}$, (3.6) reduces to the identity $\vec{\nabla} \cdot \vec{\Pi}=\nabla^{2} \Lambda=0$ and therefore defines a solution (in which $X=\Lambda$ ). Substituting the solution in (3.4) to get the energy, we find that the expression under the square root becomes a perfect square and the energy simplifies:

$$
E=\frac{1}{g_{p}} \int d^{p} x\left(1+\vec{\nabla} \Lambda^{2}\right)
$$

Note how the complicated energy function has 'linearized' and the coefficient of the quadratic term (1 instead of $1 / 2$ ) correctly reflects the sum of equal linearized energy contributions of the $X$ field and the gauge field. The net result is that the Born-Infeld theory has exactly the same BPS solutions (and not just the same energies) as the linearized theory. This is a rather stronger result than we are strictly entitled to expect. Since these solutions saturate the BPS energy bound (the energy is the string tension times the length of the strings) we expect them to be annihilated by the appropriate supersymmetries. It should be possible to check this explicitly from the formulas in [6] for the supersymmetry variations of the full Born-Infeld action, but we have yet to check this point.

There is also an interesting set of static solutions representing strings going between branes and antibranes. Branes and antibranes attract each other but for small coupling they will move towards each other very slowly so that our solutions make sense for some long time. The simplest way to find them is to exploit spherical symmetry by choosing a single-charge electric solution of the constraint and looking for the most general spherically symmetric solution of (3.6):

$$
g_{p} \vec{\Pi}=\frac{A}{r^{p-1}} \hat{r} \quad \frac{X^{\prime}\left(1+A^{2} / r^{2 p-2}\right)}{\sqrt{\left(1+A^{2} / r^{2 p-2}\right)\left(1+X^{\prime 2}\right)}}=\frac{B}{r^{p-1}} .
$$


The constant of integration $A$, since it is associated with a conserved momentum, is a conserved quantity and is related to the fundamental string tension because we are studying the electric solution (to be precise, $A=(p-2) N c_{p}$ ). The other constant of integration, $B$, is arbitrary and varying it changes the physics of the solution.

The equation for $X$ can easily be integrated to give

$$
X(r)=\int_{r}^{\infty} d r \frac{B}{\sqrt{r^{2 p-2}-r_{0}^{2 p-2}}}
$$

where we have defined a new length parameter $r_{0}^{2 p-2}=B^{2}-A^{2}$ (well-defined for $B>A$ ). This solution only makes sense for $r>r_{0}$ since $X^{\prime}\left(r_{0}\right)=\infty$, even though $X\left(r_{0}\right)$ is finite (see Figure 2b). There is a natural continuation of the surface through $r=r_{0}$, back out through increasing $r$, given by

$$
\tilde{X}(r)=2 X\left(r_{0}\right)-\int_{r}^{\infty} d r \frac{B}{\sqrt{r^{2 p-2}-r_{0}^{2 p-2}}} .
$$

This joins smoothly onto (3.9) at $r=r_{0}$ but adopts the other sign of the square root for $X^{\prime}$. The result is two parallel p-branes, joined by a throat of finite length and thickness, separated asymptotically by a distance $\Delta \equiv 2 X\left(r_{0}\right)$ (see Figure $2 \mathrm{c}$ ). The fact that one is a brane and the other an antibrane can be seen by defining an orientation on the first brane, then moving continuously to the other brane and seeing that the orientation changes.

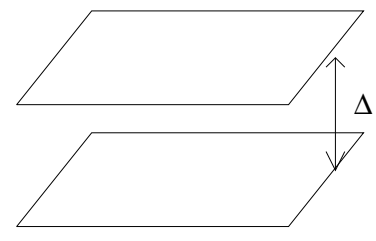

(a)

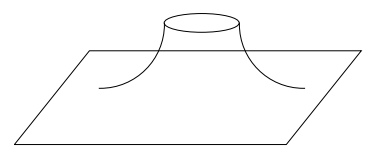

(b)

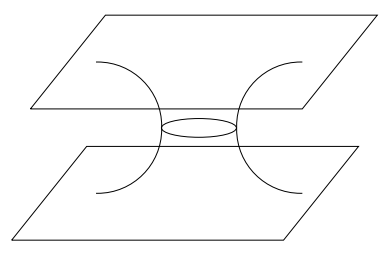

(c)

FIGURE 2:

(a) A brane-antibrane separated by a distance $\delta$, (b) Throat solution described by (3.9), (c) Full solution by patching together two solutions as in (b).

Fixing the distance between the branes $\Delta$ and the number of strings (which we do by regarding the charge parameter $\mathrm{A}$ as fixed), we have an equation to solve to fix the parameter $r_{0}$ in the solution. There are two solutions which, for large $\Delta$, are approximately

$$
r_{0} \sim A / \Delta, \quad r_{0} \sim \Delta
$$


up to a numerical constant (see Figure 3). In the first case, the radius of the throat goes to zero as $\Delta \rightarrow \infty$, a situation we interpret as a fundamental string running between the brane and the antibrane. The second solution consists of a large throat joining the branes and is easily seen to be a local maximum of the action. Note that for the second solution (Figure $3 \mathrm{~b}$ ) the electric field is a small perturbation and could be turned off without changing the solution dramatically. In the limit that the electric field is zero, it is tempting to interpret this second solution as a sphaleron associated to decay of the brane-antibrane system. Given the existence of a sphaleron, there ought to exist a 'bounce' solution describing the decay of a brane-antibrane system by tunneling under the barrier whose height is defined by the sphaleron. A bounce is a solution of the Euclidean classical equations of motion for the brane having a topology like that shown in Figure 3b (where Euclidean time would be one of the horizontal directions in the figure). Such solutions can indeed be found and describe decay of a brane-antibrane pair by nucleation of a throat joining the brane pair (followed by an expansion of the throat which converts brane worldvolume to kinetic energy of the throat expansion front). Although this is a possible picture of the decay, it is not the dominant decay mode: while the tunneling rate is of order $e^{-1 / g}$, the brane and antibrane also attract each other via long-range forces which cause them to collide and annihilate by direct string processes on a time scale of order $1 / \sqrt{g}$. Since this is much smaller than the timescale of the throat nucleation process, it seems that the tunneling is never physically meaningful 3 . One could imagine trying to take some limit of the theory where the attraction of the branes goes to zero while the tension remains finite. Such limits do not exist for branes of dimension $p \geq 1$. This is of course consistent with the fact that it has not been possible to quantize branes of dimension $p \geq 2$, if it were possible to take all distance scales to zero while keeping the brane tension finite, then we would have a situation where the branes would be fundamental.

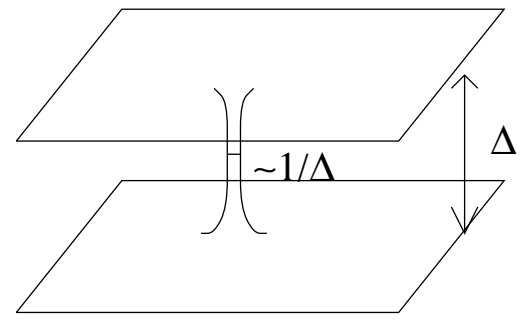

(a)

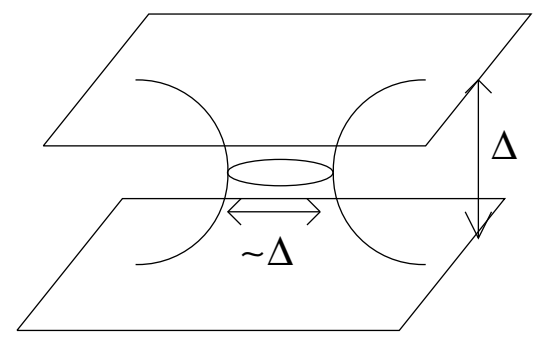

(b)

FIGURE 3: Brane - Anti-brane configuration with a throat between them.

(a) Solution corresponding to a fundamental string stretched in between.

(b) Unstable configuration.

3 We would like to thank Gary Horowitz for pointing out an error in our treatment of this point in an earlier version of this paper. 
Note that one doesn't find solutions of this type for parallel branes (as opposed to brane-antibrane). We know that parallel branes at small separation should correspond to monopoles of a spontaneously broken non-abelian worldsheet gauge theory [7]. We have not understood the exact relation between the abelian description of parallel brane-antibrane pairs and the nonabelian description of their brane-brane analog.

Although this solution makes use of the full nonlinear Born-Infeld action and is not a BPS state, it is amusing to see how close it comes to satisfying the linear BPS condition (2.1). The essential issue is the comparison of the electric field $E=F_{0 r}$ with the gradient of the scalar field, $F_{9 r}=X^{\prime}$. From the expression of the canonical momentum and the detailed form of the solution we read off that

$$
E=\frac{A}{\sqrt{r^{2 p-2}-r_{0}^{2 p-2}}}=\frac{A}{B} X^{\prime} .
$$

Substituting this result into (2.1), we find that the requirement for an unbroken susy is

$$
\left(\Gamma^{0}+\alpha \Gamma^{9}\right) \epsilon=0 \quad \alpha=A / B .
$$

Unless $A / B=1$, this is not consistent and the susy is completely broken. As we take the limit $r_{0} \rightarrow 0$, the brane pair moves further and further apart and $A / B \rightarrow 1$ (for the first solution in (3.11)). In the limit, supersymmetry and stability are recovered.

At this point, we want to say a few words about limits of validity of the solutions of Born-Infeld we have been discussing. If we replace $c_{p}$ by $N c_{p}$ in the point charge solutions, we get a solution describing $N$ superposed strings ending on a single D-brane. Since $c_{p} \sim g$ in the electric (fundamental string) case and $c_{p} \sim 1$ in the magnetic (D-string) case, we really have two parameters, $N$ and $g$, to vary in exploring the validity of our solutions. The most important region is the 'throat', roughly defined as $r \sim X^{9}$, where the transition from one type of p-brane to another takes place. Strict validity of the Born-Infeld action requires not so much that the field strengths $F_{\mu \nu}$ be small, but rather that their derivatives (measured in units of $\alpha^{\prime}$ ) be small. A bit of arithmetic on the Born-Infeld point charge solution shows that it is valid (in the sense just described) when $N c_{p} \gg 1$. Actually, to validate the neglect of gravitational effects (we have been considering branes in flat space) we also need $g^{2} N \ll 1$ in the electric case and $g N \ll 1$ in the magnetic case. As far as the throat region goes, this is all possible for large $N$ and small $g$ so long as $g \gg 1 / N \gg g^{2}$ (electric case) or $1 \gg 1 / N \gg g$ (magnetic case). Inside the throat region, toward $r=0$, fields and field gradients become large and an application of the above arguments would seem to indicate that the Born-Infeld solution can't be trusted in that regime. And yet, we have seen that a naive application of the Born-Infeld equations reproduces the correct string tension and we will soon see that more subtle features of the string dynamics are also reproduced. It seems obvious that much of what Born-Infeld tells us about the singular region is correct and that the question of limits of validity of solutions is fairly subtle. For the purposes of this paper, therefore, we will take an uncritical attitude toward this question, just to see how far we get. 


\section{S-Matrices and Boundary Conditions}

We have established that the Born-Infeld action has static solutions which correspond to strings terminating on D-branes (and also to certain D-branes terminating on higherdimensional D-objects). We have to look at the behavior of small fluctuations propagating on our static solutions with special attention to how they reflect from (or transmit through) the effective junction between two kinds of brane. As we shall see, it is here that the specific nonlinearities of the action become quite crucial.

We take the BPS solution for a string attached to a 3-brane as background and, for simplicity, study the propagation of perturbations of a scalar field describing motion in a direction perpendicular to both branes. We denote the fluctuation coordinate by $\eta$ and look at s-wave fluctuations in the obvious spherical coordinate system. The linearized small fluctuation expansion of the Born-Infeld action about the exact background turns out to be

$$
-\left(1+\frac{g^{2} c^{2}}{r^{4}}\right) \partial_{t}^{2} \eta+r^{-2} \partial_{r}\left(r^{2} \partial_{r} \eta\right)=0
$$

The entire effect of the nonlinearities is contained in the $r^{-4}$ term multiplying the $\partial_{t}^{2}$ : apart from that, the equation is just the linear $3+1$-dimensional D'Alembertian. For future interpretation, recall that the field giving the static transverse displacement of the 3 -brane is $X^{9}=g c / r$. In the limit $r \rightarrow \infty$, the extra term can be neglected, and one has the usual interpretation of spherical waves freely propagating in three dimensions. One might have thought that the $r^{-4}$ term just acts as a potential from which the otherwise free waves scatter. In fact, it is so singular that the usual interpretation does not work and $r \rightarrow 0$ functions as a second asymptotic regime to which waves can escape. To see this, drop the constant term in the coefficient of $\partial_{t}^{2} \eta$ in (4.1) and make the change of variables $w=g c / r=X^{9}(r)$. The result is very simple:

$$
\left(-\partial_{t}^{2}+\partial_{w}^{2}\right) \eta=0
$$

the equation for free propagation in one dimension (at the correct velocity of light)! This is the correct dynamical counterpart to the identification of the static background solution as a string attached to a threebrane. It relies in an essential way on the specific nonlinear structure of the Born-Infeld action and, of course, explores almost, but not exactly, BPS physics.

In order to fully verify the correspondence with Polchinki's picture of D-brane dynamics via open fundamental strings, we have to look into the effective boundary condition for small fluctuations on the string (the $r \rightarrow 0$ region) imposed by the presence of the three-brane (the $r \rightarrow \infty$ region). In other words, we want the S-matrix connecting the two asymptotic regions. We can get most of the information we want via the following 
sequence of arguments. We first reduce to a purely radial problem by projecting (4.1) on frequency $\omega$ :

$$
\left(\frac{d^{2}}{d x^{2}}+1+\frac{\kappa^{2}}{x^{4}}\right) \eta=0 \quad x=\omega w=\frac{\omega g c}{r} \quad \kappa=g c \omega^{2} .
$$

This shows that the problem has an interesting joint dependence on string coupling, string tension and energy. Then we make a coordinate change suggested by WKB considerations 4 :

$$
\xi(x)=\int_{\sqrt{\kappa}}^{x} d y \sqrt{1+\frac{\kappa^{2}}{y^{4}}} \quad \eta=\left(1+\frac{\kappa^{2}}{x^{4}}\right)^{-1 / 4} \tilde{\eta} .
$$

This transforms $(4.3)$ to

$$
\left(-\frac{d^{2}}{d \xi^{2}}+V(\xi)\right) \tilde{\eta}=\tilde{\eta} \quad V(\xi)=\frac{5 \kappa^{2}}{\left(x^{2}+\kappa^{2} / x^{2}\right)^{3}}
$$

where $\xi$ now runs over the full real line while the potential $V(\xi)$ vanishes as $\xi \rightarrow \pm \infty$ and is non-zero, but finite, in the region of $\xi \sim 0$. Consequently, in the new variables, we have a perfectly conventional one-dimensional scattering problem.

A closer look at how the potential scales shows that, in various limits, we may make the replacements

$$
\begin{aligned}
& V(\xi) \sim \frac{a}{\sqrt{\kappa}} \delta(\xi) \quad \kappa \rightarrow 0 \\
& V(\xi) \sim 0 \quad \kappa \rightarrow \infty
\end{aligned}
$$

where $a$ is a dimensionless constant. We are interested in the behavior of the onedimensional reflection and transmission amplitudes $R$ and $T$. Simple considerations about delta-function potentials show that

$$
\begin{array}{rlrl}
R \rightarrow-1 & T \rightarrow 2 i \sqrt{\kappa} / a & \text { as } \quad \kappa \rightarrow 0 \\
R \rightarrow 0 & T \rightarrow 1 & \text { as } \quad \kappa \rightarrow \infty
\end{array}
$$

and we could, if we wanted, learn more about the energy variation of these amplitudes.

We can now make contact with the Polchinski picture of D-branes as boundaries for open strings. Since $\tilde{\eta}$ is a disturbance on the string attached to the threebrane, transverse to both the brane and the string, it should, on Polchinski's picture, satisfy a Dirichlet (fixed) boundary condition. In terms of our one-dimensional scattering theory picture, this amounts to requiring $R=-1$ (and $T=0$ ). Our analysis of the scattering problem shows that this is indeed the low-energy limit of the S-matrix but that, as the energy increases, the S-matrix goes over from perfectly reflecting to perfectly absorbing. The

4 We want to thank K. Saviddi for assistance on this point. 
energy scaling of (4.3) indicates that the characteristic energy of the transition between the two behaviors is $\omega_{c r i t} \sim 1 / \sqrt{g c}$. The parameter $c$ is related to the string tension and we find that, for the fundamental (electric) string case, $\omega_{\text {crit }} \sim 1 / \sqrt{g \alpha^{\prime}}$. While, for the D-string (magnetic) case $\omega_{\text {crit }} \sim 1 / \sqrt{\alpha^{\prime}}$. In the weak-coupling $g \rightarrow 0$ limit, the Dirichlet boundary condition accurately describes the scattering physics of the fundamental open string at large energies compared to the string scale and therefore presumably captures the essential string theory dynamics. On the other hand, in the magnetic or D-string case, the Dirichlet boundary condition only describes the physics below the string scale and thus only captures the field theory limit of the problem.

\section{Higher dimensional solitons}

In previous sections we considered excitations of the branes which included only one transverse field. Now we consider excitations involving two transverse fields (transverse positions). While the solutions in this section are well known [8,9, 10, 11, we believe that we will present them under a new and interesting light. We also want to constrast them with our previous discussion.

Consider a two brane. In fact one can consider any relativistic brane (a D-brane, M-brane, or even a fundamental string in Euclidean space) but just concentrate on two of its spatial coordinates. Denote by $z=X^{1}+i X^{2}$ two of the worldvolume coordinates and let $w=X^{3}+i X^{4}$ be two of the transverse coordinates. All the nontrivial dynamics will involve only the $(z, w)$ variables. The linear equation for fluctuations of $w$ is just the laplacian $\partial_{z} \partial_{\bar{z}} w=0$. A solution to this equation is to take $w(z, \bar{z})$ to be holomorphic (i.e. $w(z))$. A crucial point is that a holomorphic function is a solution of the full Nambu action for the brane and, in the supersymmetric case, these holomorphic solutions are also BPS [8,10]. This can be recognized as the usual condition for worldsheet instantons for the case of fundamental strings. One interesting solution is $w=c / z$, where $c$ is a constant. We see that as we approach $z \rightarrow 0$ we sweep out the $w$ plane, so near the origin the surface is really another brane stretched along the $w$ plane. A pair of D2 branes intersecting at a point is represented by $z w=0$ so that the deformation $z w=c$ is the single smooth surface you get by blowing up the intersection point between the original pair of D2 branes into a smooth finite throat. In the D-brane case (reinstating the full BornInfeld action) there can also be a gauge field. Any pure gauge field (holomorphic vector potential and vanishing field strength) is in principle acceptable, but the configuration $A_{z}=A_{z}^{\infty}+a / z-A_{w}^{\infty} c / z^{2}$ is the only one for which $A_{z}$ stays finite as $z \rightarrow \infty$ and the transformed field $A_{w}=\frac{\partial z}{\partial w} A_{z}=A_{w}^{\infty}-a / w-A_{z}^{\infty} c / w^{2}$ stays finite as $w \rightarrow \infty$. The constants $A_{z}^{\infty}, A_{w}^{\infty}$ are the Wilson lines that would be present on two non-intersecting D2 branes. The extra parameter, $a$, is a new Wilson line or modulus which is only allowed 
when the branes intersect. Since $a$ is complex, there are two new real moduli. One of them is measured by integrating the vector potential along a contour which circles the origin of the $z$ plane and the other is measured by a contour which goes through the throat and emerges in the $w$ plane (this last one would be finite if the branes were finite). Overall, there are four extra real parameters characterizing the deformation: the two complex constants $c, a$. If the constant $c$ in the equation $z w=c$ is much bigger than $\alpha^{\prime}$, then we can trust the solution. Notice that in this case, as opposed to the case discused in sections 2 and 3, we do not need to consider a large number of branes to trust the solution, we can just tune a parameter in the solution. Obviously, in the case of M-branes the condition is that $c \gg l_{11}^{2}$.

It is an amusing exercise to see how this description arises from the point of view of D-brane probes [12,13]. To that effect it is convenient to add two more dimensions so that we are describing two D4 branes intersecting along two dimensions. So we have a 4-brane along $(z, t)$ and another 4-brane along $(w, t)$ where $t$ is another complex coordinate describing a two dimensional plane. Then we can consider a D0 brane probe moving in this geometry [14]. Starting from the singular configuration we can write a Lagrangian involving $(0,4),\left(0,4^{\prime}\right)$ and $\left(4,4^{\prime}\right)$ strings. This action will have four real supersymmetries and can be viewed as the dimensional reduction of a 4 dimensional $N=1$ action. In fact the four dimensional action describes a D3 brane probe in the presence of two orthogonal D7 branes of the same $(p, q)$ type. We can therefore use four dimensional $N=1$ superfield notation. We will be interested in the superpotential

$$
S_{p o t}=\int d t d^{2} \theta\left\{z_{00} \phi_{04}^{+} \phi_{04}^{-}+\omega_{00} \phi_{04^{\prime}}^{+} \phi_{04^{\prime}}^{-}+\phi_{44^{\prime}}^{+} \phi_{04^{\prime}}^{+} \phi_{04}^{-}+\phi_{44^{\prime}}^{-} \phi_{04^{\prime}}^{-} \phi_{04}^{+}\right\}+c . c .
$$

where $z_{00}, w_{00}$ are the chiral superfields associated to the position of the zero brane in the $z$ and $w$ plane. $\phi_{04}^{ \pm}$are two chiral superfields associated to the $(0,4)$ strings and $\phi_{04^{\prime}}^{ \pm}$ are associated to $\left(0,4^{\prime}\right)$ strings. Finally $\phi_{44^{\prime}}^{ \pm}$are associated to $\left(4,4^{\prime}\right)$ strings. We now fix $\phi_{44^{\prime}}^{ \pm}, z_{00}, w_{00}$ and we diagonalize the superpotential for the 04 and $04^{\prime}$ fields. After we do this we get new fields with holomorphic mass parameters

$$
m_{ \pm}=\frac{z_{00}+w_{00}}{2} \pm \sqrt{\left(\frac{z_{00}+w_{00}}{2}\right)^{2}-\phi_{44^{\prime}}^{+} \phi_{44^{\prime}}^{-}}
$$

If we fix the value of the 44 ' strings we find that there are some values of $z_{00}, w_{00}$ for which we have a massless mode. Physically this means that the 0-brane probe is touching the fourbrane. We see that $m_{ \pm}=0$ implies that $z_{00} w_{00}=\phi_{44^{\prime}}^{+} \phi_{44^{\prime}}^{-}$, this is the same equation as we found before for the shape of the single (in this case) fourbrane. We see that exciting the 44' strings corresponds to a complex deformation (some "blow up") of the two intersecting 4 branes into a smooth configuration. 
Another amusing exercise is to take one of the transverse fields to be compact, $X^{4} \sim$ $X^{4}+2 \pi$ to be specific. In this case $w=\log z$ is a solution. This solution represents a membrane wrapped around the $\hat{4}$ direction, lying along $\hat{5}$ and ending on a two brane oriented along 23. This is how a fundamental string ends on a D2 brane from the point of view of M-theory [15]. This is vortex solution since the field $X^{4}$ winds once when we go once around the origin. This type of configuration (with some more branes) was used to solve $\mathrm{N}=2$ gauge theories in four dimensions in [16].

These results can be used to count the entropy of black holes [11]. Suppose that we consider a system to $Q_{3}$ threebranes oriented along 789, $Q_{3}^{\prime}$ D-threebranes oriented along 569. Then we can say that the $z$ plane is associated to 56 and the $w$ plane to 78 . We can see that we have $Q_{3} Q_{3}^{\prime}$ intersection points. At each of those points we can make the deformation described above. This introduces $4 Q_{3} Q_{3}^{\prime}$ bosonic parameters, and an equal number of fermionic ones. Which then give a CFT with central charge $c=6$ along the direction 9, producing the right black hole entropy [11. So the black hole entropy comes from deforming the D3-brane "foam" that results after "blowing up" each of the intersection points. This is similar to saying that the entropy comes from 44' strings [17]. Similarly, if we replace the $T^{4}$ along 5678 with a K3 we then the threebranes will be some holomorphic two-surface on K3 with $c_{I J} p^{I} p^{J} / 2$ intersection points ( $p^{I}$ are the vectors specifying the charge configuration) and the counting of the entropy proceedes in the same way [11].

\section{Conclusions}

We have found some solutions of the Born-Infeld action describing strings ending on D-branes, or M2-branes ending on M5-branes. It would be interesting to see if one can pursue a bit further this idea that lower-dimensional branes arise from higher-dimensional ones. We also found solutions describing a string going between a brane and an anti-brane. We studied the propagation of small perturbations on these solutions and found that the cross sections had the properties required to understand Polchinski's picture of D-branes as boundaries of open strings. We also see that when we have a brane-antibrane pair we can, in some sense, interpret electric charges as some kind of microscopic "wormholes" going from one brane to the other in a purely geometric fashion. This realizes Wheeler's old idea of 'charge without charge' (the existence of both electric and magnetic charges provides the charge quantization condition).

\section{Acknowledgements}

We would like to thank G. Horowitz for pointing out problems in an earlier version of this paper related to our interpretation of the Euclidean bounce solution as a decay mode of a brane-antibrane pair. 
We would like to thank M. Douglas, D. Lowe and J. Schwarz for interesting discussions and for informing us about their work in this area [18]. Also, after completing this work, we learned of a study of related matters by Gibbons et al [19]. We also thank M. Kroyter for pointing out a couple of typos.

C.G.C. and J. M. were supported in part by DOE grants DE-FG02-91ER40671 and DE-FG02-96ER40559 respectively. 


\section{References}

[1] J. Polchinski, Tasi Lectures on D-branes, hep-th/9611050.

[2] R. Leigh, Mod. Phys. Lett .A4 (1989) 2767.

[3] A. Strominger, Phys .Lett .B383 (1996) 44, hep-th/9512059.

[4] P. Townsend, Phys. Lett. B373 (1996) 68, hep-th/9512062.

[5] J.M. Maldacena and A. Strominger, Phys. Rev. Lett. 77 (1996) 428, hep-th/960.

[6] M. Aganagic, C. Popescu and J. Schwarz, Nucl. Phys. B495 (1997) 99, hep-th/9612080.

[7] D. Diaconescu, D-branes, Monopoles and Nahm Equations, hep-th/9608163.

[8] X. Wen and E. Witten, Phys. Lett. 166B (1986) 397.

[9] C. Vafa, M. Bershadsky, C. Vafa and V. Sadov, Nucl. Phys. B463 (1996) 420, hepth/9511222; Nucl. Phys. B463 (1996) 398, hep-th/9510225.

[10] K. Becker, M. Becker and A. Strominger, Nucl. Phys. B456 (1995) 130, hepth/9507158.

[11] A. Strominger and C. Vafa, Phys. Lett. B379 (1996) 99, hep-th/9601029.

[12] M. Douglas and G. Moore, D-branes, quivers and ALE instantons, hep-th/9603167.

[13] M. Douglas, Gauge fields and D-branes, hep-th/9604198.

[14] J. Maldacena and A. Strominger, unpublished notes.

[15] O. Aharony, J. Sonnenschein and S. Yankielowicz, ucl. Phys. B474 (1996) 309, hepth/9603009.

[16] E. Witten, Solutions of four dimensional field theories via M-theory, hep-th/9703166.

[17] C.G. Callan and J.M. Maldacena, Nucl. Phys. B472 (1996) 591, hep-th/9602043.

[18] M. Douglas, D. Lowe and J. Schwarz, private comunication.

[19] Lecture at Centro de Estudios Cientificos de Santiago, August 1997. 